\title{
Polarization-based Transparent Surface Modeling from Two Views
}

\author{
Daisuke Miyazaki*
}

\author{
Masataka Kagesawa $^{\dagger}$ \\ The University of Tokyo, Japan \\ http: //www.cvl.iis.u-tokyo.ac.jp/
}

\author{
Katsushi Ikeuchi ${ }^{* \dagger}$
}

\begin{abstract}
In this paper, we propose a novel method to recover the surface shape of transparent objects. The degree of polarization of the light reflected from the object surface depends on the reflection angle which, in turn, depends on the object's surface normal; thus, by measuring the degree of polarization, we are able to calculate the surface normal of the object. However, degree of polarization and surface normal does not correspond one-to-one, making us to analyze two polarization images taken from two different view in order to solve the ambiguity. A parabolic curve will be a strong clue to correspond a point in one image to a point in the other image, where both points represent the same point on object surface. By comparing the degree of polarization at such corresponding points, the true surface normal can be determined.
\end{abstract}

\section{Introduction}

In the field of computer vision, various methods have been developed to deal with opaque object. However, few methods focus on transparent object. Environment matting $[1,2]$ is developed to synthesize a realistic image of transparent object without using any information about the 3D shape of the object. Schechner et al. [3] and Szeliski et al. [4] separated the overlapped image of glass plates into two images; one is the reflected image, while the other is the transmitted image. Murase [5] proposed a method to determine the surface shape of water wave by analyzing the image placed in the bottom of the water, which causes the image to be deformed due to refraction and undulation. Hata et al. [6] projected a light stripe onto transparent objects and recovered the surface shape of transparent objects by applying a genetic algorithm.

Shape-from-polarization is known to be effective to estimate the shape of specular objects such as metals or transparent objects. Koshikawa et al. [7,8] analyzed the polar-

\footnotetext{
*Department of Computer Science, Graduate School of Information Science and Technology

${ }^{\dagger}$ Interfaculty Initiative in Information Studies, Graduate School of Interdisciplinary Information Studies

† This research was supported in part by Japan Science and Technology Corporation under the Ikeuchi CREST project.
}

ization of specular objects to retrieve, from the database, a 3D model of an object which matches the observed object. Wolff et al. $[9,10]$ attempted to determine the surface shape of objects by using a combined method of polarization analysis and binocular stereo; however, they did not propose a method to search the corresponding points of two images; thus, they measured only the orientation of the glass plane and the metal plane. Saito et al. [11] tried to measure the surface shape of transparent objects; unfortunately, there was an ambiguity problem for determining the true surface normal, and they simply heuristically solved the ambiguity. Miyazaki et al. [12] extended Saito's method and solved the ambiguity problem from a polarization analysis of thermal radiation, and determined the surface shape of transparent objects. Rahmann et al. [13] proposed an optimization method, and measured the surface shape of an opaque specular sphere by using polarization images taken from 5 directions. Recently, Miyazaki et al. [14] also proposed a method to estimate the surface shape of an opaque specular object from a polarization image from a single view.

In this paper, we propose a method to determine the surface shape of transparent objects by using a polarization phenomena of reflected light. We solve the ambiguity problem of surface normal in Saito et al.'s method by rotating the object at a small angle, and also solve the corresponding problem of binocular stereo in Wolff et al.'s method by using the geometrical property of the object surface. We do not require camera calibration, so we do not need to know the position of a projector as in Hata et al.'s method. Moreover, we do not need to know the rotation angle for stereo as in Rahmann et al.'s method. We use parabolic curves [15-17], curves whose Gaussian curvature are 0, for searching the corresponding points.

Section 2 explains about the polarization analysis, especially, describing the ambiguity problem of surface normal. The relationship between the degree of polarization and the reflection angle is not one-to-one, so that one cannot determine the true surface normal unless one solves this ambiguity problem.

In Section 3, we describe the method to solve the ambigutiy problem. The disambiguation is done by introducing a binocular stereo method, practically, we rotate the ob- 


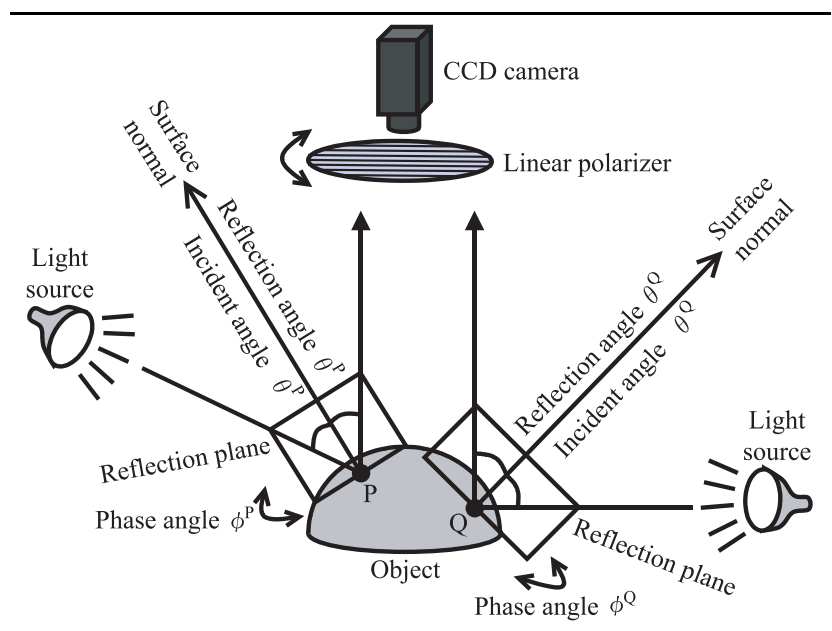

Figure 1. Location of acquisition system

ject at a small angle instead of setting two cameras. We compare two polarization images taken from two different directions: one polarization image is taken before the object is rotated and the other polarization image is taken after the object is rotated at a small angle. The degree of polarization is compared at a pair of points which correspond to an identical point on the object surface. The corresponding method is explained by using a Gaussian sphere.

We present an experimental result in Section 4, and suggest topics for our future work in Section 5.

\section{DOP and reflection angle}

In this section, we describe the relationship between the degree of polarization (DOP) and the reflection angle. For details, please refer to $[9-12,18]$.

Geometrical location of the acquisition system is shown in figure 1 . We locate the polarizer-mounted camera over the target object, and illuminate the object with unpolarized light traveling through the air, whose refractive index is 1.0. Unpolarized light will become partially polarized if the light reflects from the object surface, whose refractive index is $n$. We observe such reflected light by the polarizer-mounted camera. Note that, in our analysis, we use an orthographic projection assumption.

The angle between the light source direction and the surface normal is called the incident angle, and the angle between the surface normal and the camera direction is called the reflection angle. The incident angle is equal to the reflection angle for optically smooth transparent objects. The plane consisting of the surface normal and the camera direction is called the reflection plane. Consider projecting the reflection plane onto the image plane: the reflection plane appears to be a straight line on the image plane, and the orientation of this line is called a phase angle.

Surface normal can be represented by zenith angle $\theta$ and azimuth angle $\phi$ in polar coordinate system. Reflection

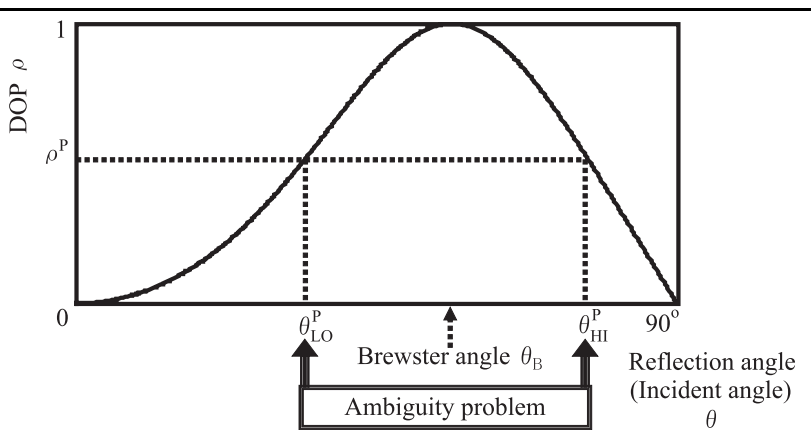

Figure 2. DOP and reflection angle $(n=1.5)$

angle (= incident angle) corresponds to zenith angle, and phase angle corresponds to azimuth angle. Two phase angles are calculated in the range of $0 \leq \phi<360^{\circ}$, and the difference of those two angles is $180^{\circ}$. Since we know the surface normal is heading vertically to the viewing direction at the occluding boundary, we can solve the ambiguity problem of the phase angle by propagating the determination of the phase angle from the occluding boundary to the inner part of the object region. Therefore, we have only to determine the reflection angle in order to obtain the surface normal of the object surface.

We rotate the polarizer and observe the object by the camera and calculate the DOP, which for reflected light ranges from 0 to 1: DOP is 0 for unpolarized light, and DOP is 1 for perfectly polarized light. The following formula represents the relationship between DOP and the reflection angle;

$$
\rho=\frac{2 \sin ^{2} \theta \cos \theta \sqrt{n^{2}-\sin ^{2} \theta}}{n^{2}-\sin ^{2} \theta-n^{2} \sin ^{2} \theta+2 \sin ^{4} \theta} .
$$

DOP $\rho$ is the function of refractive index $n$ and reflection angle $\theta$. We assume that the refractive index is given, and compute the reflection angle from the DOP.

Figure 2 illustrates equation (1). The vertical axis represents the DOP, and the horizontal axis represents the reflection angle. If the reflection angle is $0^{\circ}$ or $90^{\circ}$, then the DOP will be 0; and if the reflection angle is Brewster angle, $\theta_{B}$, then the DOP will be 1 . We obtain two reflection angles from one DOP, except for the Brewster angle. One of the angles is the true reflection angle and the other is not. Our task is to solve this ambiguity problem to determine the true surface normal.

\section{Disambiguation}

First, the DOP data will be segmented and classified into three types of region, as described in Section 3.1. Next, we prove the folding curve, defined in Section 3.1, is a geometrical property of object surface, in Section 3.2. Then, we rotate the object to obtain the DOP data from two different views. Section 3.3 describes the method to search corresponding points for the pair of DOP data by using the 


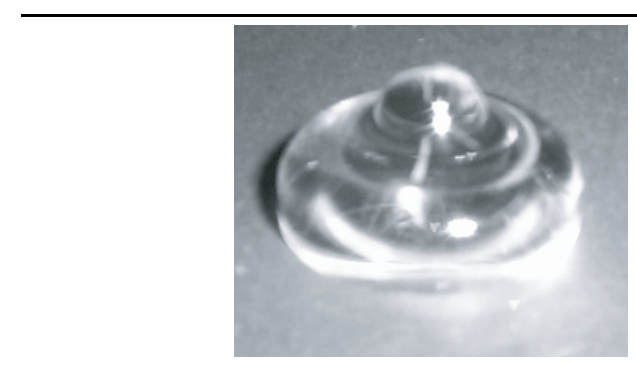

Figure 3. Photograph of bell-shaped acrylic object

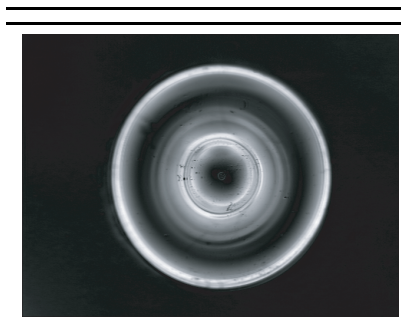

(a)

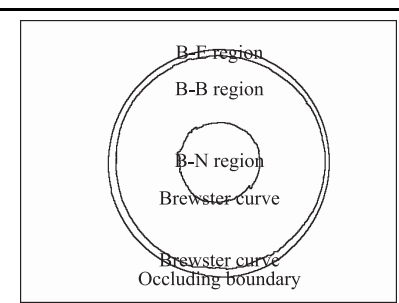

(b)
Figure 4. (a) DOP image, (b) result of Brewster segmentation; of bell-shaped acrylic object

folding curve. Finally, the method described in Section 3.4 solves the ambiguity problem by comparing the DOP at corresponding points.

\subsection{Region segmentation}

We calculate the DOP values of all of the points over the object surface, and call the set of those DOP values the DOP image. Figure 3 is a photograph of a bell-shaped transparent object, and DOP image of this object is shown in figure 4(a). The DOP is represented as a gray image in figure 4(a), where black represents $0 \mathrm{DOP}$ and white represents $1 \mathrm{DOP}$.

We divide the DOP image into some regions whose boundaries will be the Brewster curve, the closed curve which consists of only Brewster angle (= 1 DOP). We call this segmentation method a Brewster segmentation method. We assume the target object to have a closed smooth surface, $C^{\infty}$, thus, we can disambiguate all of the points in the region even if we disambiguate only one point in the region.

Figure 4(b) is the result of Brewster segmentation computed from the DOP image shown in figure 4(a). There are 2 Brewster curves and 1 occluding boundary in figure $4(\mathrm{~b})$.

We classify each region into 3 types (figure 5):

1. B-E region

2. B-N region

3. B-B region

$\mathrm{B}$ is for Brewster, $\mathrm{N}$ is for North pole, and $\mathrm{E}$ is for Equator.

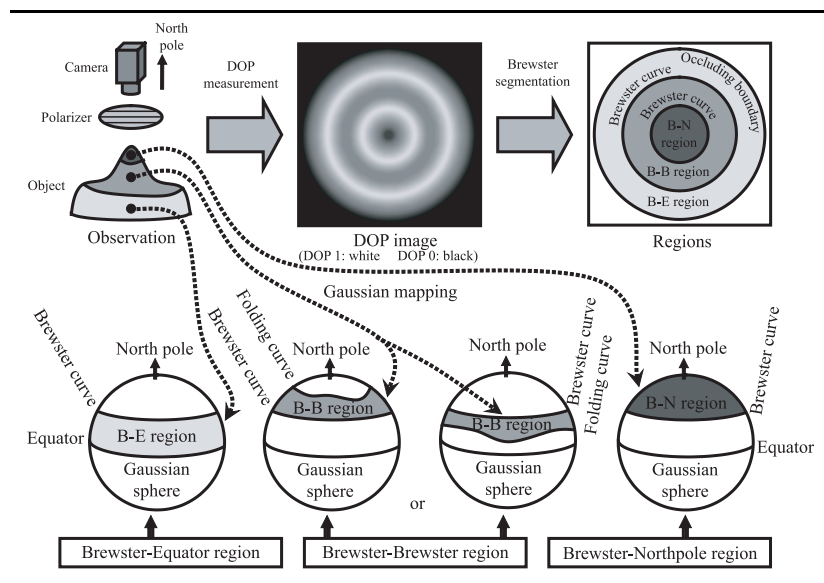

Figure 5. Gaussian mapping and regions

There is one B-E region, B-N region, B-B region each in figure 4(b).

Figure 5 represents the Gaussian mapping of regions from object surface to the Gaussian sphere. The north pole of the Gaussian sphere represents the camera position.

We define the B-E region as a region that includes an occluding boundary. The occluding boundary will be mapped onto the equator of the Gaussian sphere, whose points satisfy $\theta=90^{\circ}$. We can disambiguate the points of B-E region as $\theta_{B} \leq \theta \leq 90^{\circ}$. The occluding boundary is calculated from background subtraction.

We define $\mathrm{B}-\mathrm{N}$ region as a region including a point where $\theta=0^{\circ}$. Points where $\theta=0^{\circ}$ will be mapped onto the north pole of Gaussian sphere. We assume that there is no selfocclusion although we rotate the object at a small angle, namely, if there is a point where $\rho=0$ in the region and there is still a point with $\rho=0$ in the region even if we rotate the object at a small angle, then we know that such point is not $\theta=90^{\circ}$ but $\theta=0^{\circ}$. Therefore, we can disambiguate the points of $\mathrm{B}-\mathrm{N}$ region as $0^{\circ} \leq \theta \leq \theta_{B}$.

We define $\mathrm{B}-\mathrm{B}$ region as a region which is not $\mathrm{B}-\mathrm{E}$ region nor B-N region; thus, B-B region on the Gaussian sphere does not include the north pole nor the equator.

\subsection{Folding curve and parabolic curve}

Since the object surface is smooth, a closed region on the object surface maps onto a closed region on the Gaussian sphere. Therefore, B-B region on Gaussian sphere is always enclosed by a Brewster curve and other additional curve(s). We define such a curve, which is not a Brewster curve, as a folding curve on the Gaussian sphere. From the theorem provided below, we can conclude a folding curve to be a geometrical property of the object surface which is useful for searching corresponding points.

Theorem 1 A folding curve on the object surface is a parabolic curve. 


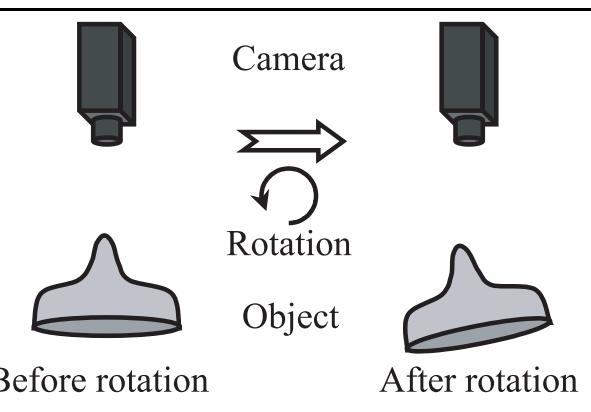

Figure 6. Object rotated at a small angle

Proof. Surface can be represented as $f(x, y)$, and the surface normal can be represented as $\mathbf{n}=(-p-q 1)^{T}$ where $p=\partial f / \partial x$ and $q=\partial f / \partial y$. Gaussian sphere is a representation of a surface normal. Let $P$ be a point on the folding curve in the Gaussian sphere, and $Q$ be a point on the object surface such that $g(Q)=P$. Then, according to the definition of a folding curve, in neighbourhood of $Q, g$ is not homeomorphic, in other words, $Q$ is a critical point of $g=\left(f_{x}, f_{y}\right)$, thus, $\nabla p=\nabla q=0$. Gaussian curvature $K$ will be;

$$
K=\frac{\operatorname{det}\left(\begin{array}{cc}
\frac{\partial^{2} f}{\partial x^{2}} & \frac{\partial^{2} f}{\partial x \partial y} \\
\frac{\partial^{2} f}{\partial y \partial x} & \frac{\partial^{2} f}{\partial y^{2}}
\end{array}\right)}{E G-F^{2}}=0,
$$

where $\left(\begin{array}{ll}f_{x x} & f_{x y} \\ f_{y x} & f_{y y}\end{array}\right)$ is the Hessian. $E, F$ and $G$ are the parameters of the first fundamental form [15] where $E G-$ $F^{2}>0$ holds.

\subsection{Corresponding point}

We rotate the object against the camera at a small angle (figure 6). We solve the ambiguity problem by comparing DOP images taken both before and after rotation. Two DOP values must be compared at an identical point on the object surface. We have to search for two points, one point in each DOP images, where geometrical properties of the object surface coincide.

Consider a great circle on Gaussian sphere which represents the rotation direction. We call this great circle the rotation circle. The rotation circle includes the two poles. Points that map onto the rotation circle still map onto the rotation circle after the object rotation. Surface normal of the points on the rotation circle is parallel to the rotation direction. We define the intersection of the rotation circle and the global folding curve of B-B region as the corresponding point. From figure 7, we realize that the corresponding point is a point which has minimum DOP within the points in $\mathrm{B}-\mathrm{B}$ region where the surface normal is parallel to the rotation direction.

We first execute Brewster segmentation to the obtained two DOP images, and then we search for one corresponding point in each B-B region. If we have no corresponding point

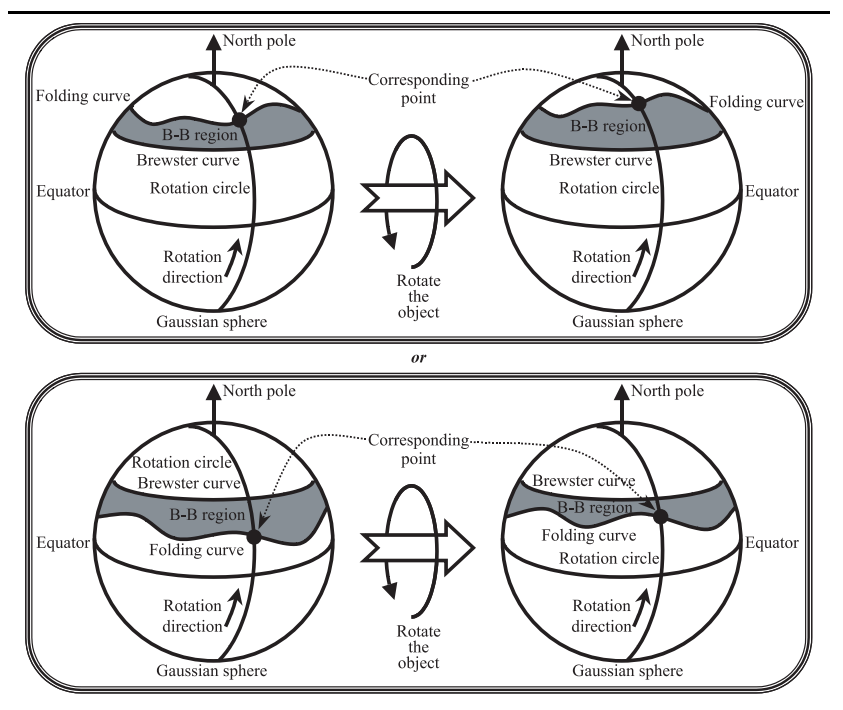

Figure 7. Corresponding point

in a certain rotation direction, we rotate the object in many directions to obtain the corresponding point.

\subsection{Difference of DOP}

We represent the DOP of the object before rotation as $\rho(\theta)$, and represent the DOP of the object rotated at a small angle $\Delta \theta$ as $\rho(\theta+\Delta \theta)$. Then, the difference of the DOP at a pair of corresponding points will be as follows;

$$
\rho(\theta+\Delta \theta)-\rho(\theta) \simeq \rho^{\prime}(\theta) \Delta \theta .
$$

The graph of DOP $\rho$ is shown in figure $8(\mathrm{a})$, and the graph of the derivative of DOP $\rho^{\prime}$ is shown in figure 8(b).

The derivative of the DOP is positive when $0^{\circ}<\theta<$ $\theta_{B}$, and negative when $\theta_{B}<\theta \leq 90^{\circ}$. We assume that the rotation direction is given, namely, the sign of $\Delta \theta$ is given. The sign of the difference of the DOP at corresponding points can be calculated from obtained DOP images. Thus, from equation (3), we can determine whether the points of the region satisfy $0^{\circ}<\theta<\theta_{B}$ or $\theta_{B}<\theta \leq 90^{\circ}$; namely, we can solve the ambiguity problem.

Our proposed method uses only the sign for disambiguation, not the value. We do not need to know the rotation angle - the absolute value of $\Delta \theta-$ so that camera calibration is not needed. Even if there was an error in DOP value or even if the rotation angle was not so small or even if we do not give a precise rotation direction, the sign of DOP would rarely change, thus, the method can be considered to be robust.

\section{Experiments}

\subsection{Experimental method}

Our acquisition system is described in figure 9. As a light source, we employ a spherical diffuser illuminated from three 300-W incandescent light bulbs, located circu- 


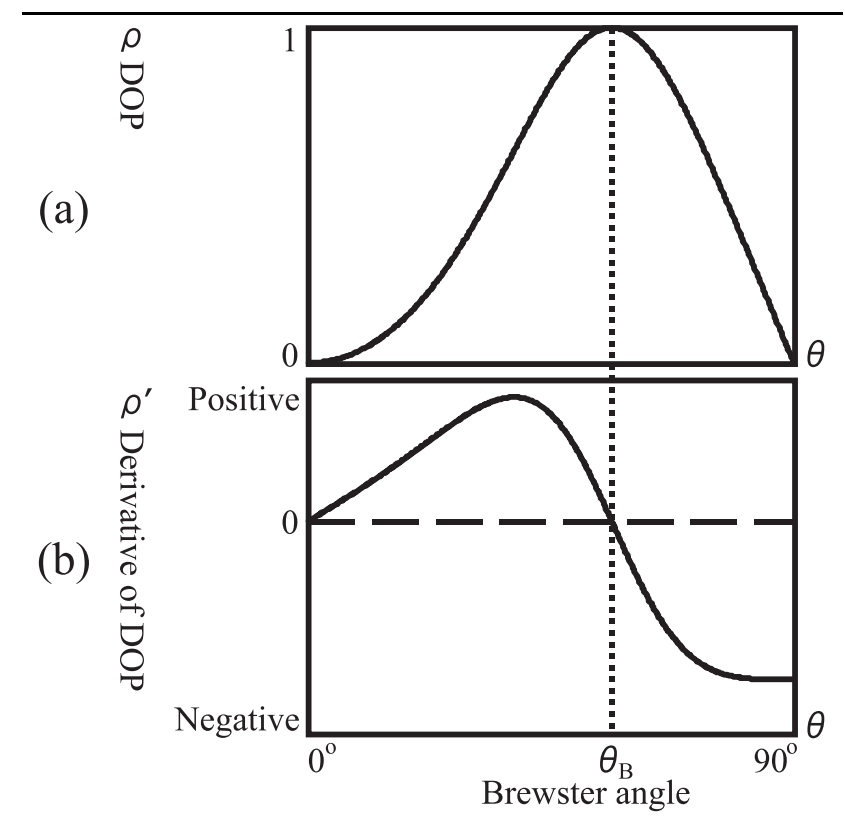

Figure 8. (a) DOP, (b) derivative of DOP; $(n=$ $1.5)$

larly 120 degrees apart. The spherical diffuser is made of plastic, and its diameter is $40 \mathrm{~cm}$. This spherical diffuser becomes an unpolarized spherical light source and illuminates an object that is located at the center of the sphere from all directions. Because we determine surface orientations using only surface reflection and the surface reflection occurs only when the reflecting and incident angles are the same, it is necessary to illuminate an object from all directions in order to observe surface reflections over the entire object surface.

The object is observed through a small hole at the top of the sphere by a monochrome CCD camera. A polarization filter is mounted between the hole and the camera.

We set a black plastic pipe on a black cloth and set the object on the pipe to avoid the light emitting from behind the object. However, interreflections still occur, reflecting many times between the front-facing surface and backfacing surface. Since we cannot know the object shape a-priori, we cannot perfectly estimate such interreflection. Thus, we assume the light caused by the interreflection is an uniform unpolarized light. We estimate the intensity of such light and we previously subtract the intensity from input images.

We calculated the DOP by fitting a sinusoid with leastsquare method from 36 images whose orientation of the polarizer was from $0^{\circ}$ to $175^{\circ}$ at intervals of $5^{\circ}$. We obtained DOP images of the object surface by the acquisition system, computed the surface normal of object surface, and finally integrated the surface normal data to the height data by us-

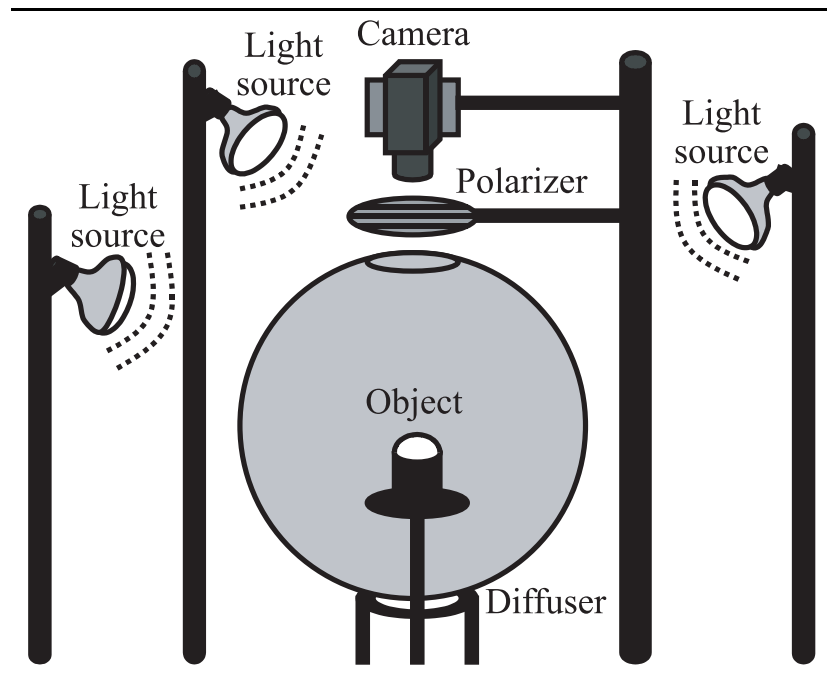

Figure 9. Experimental setup

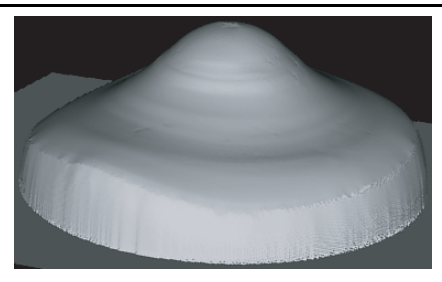

(a)

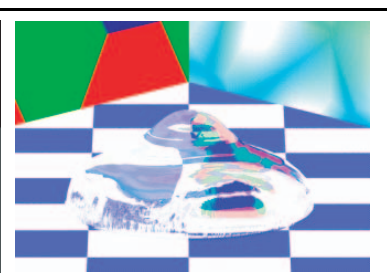

(b)
Figure 10. Obtained shape of acrylic bellshaped object: (a) shading image, (b) raytracing image

ing relaxation method $[16,19]$.

\subsection{Experimental result}

First we measured an acrylic transparent hemisphere whose refractive index is 1.5 and diameter is $30 \mathrm{~mm}$. Height error for this hemisphere was $1.1 \mathrm{~mm}$. The height error is an average error throughout the entire object surface, computed as an absolute difference between the true height and the obtained height.

Next, we applied our method to the object shown in figure 3. The target object was a bell-shaped acrylic object where the refractive index is 1.5 and the diameter(width) was $24 \mathrm{~mm}$. We computed the entire surface shape of the object by our method. The rendered images are shown in figure 10: figure 10(a) is an example of shading image, figure $10(b)$ is an example of raytracing result.

Figure 11 illustrates the shape of the object observed from the side view. The obtained shape is represented as dotted curve, and the true shape is represented as solid curve. Note that we artificially created the true shape by hand using the silhouette from the photograph of the object. 


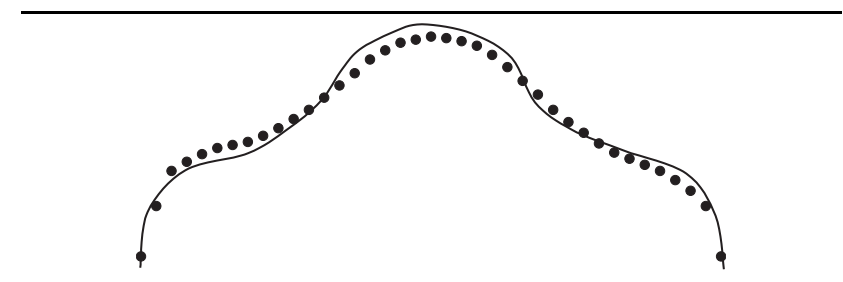

Figure 11. Obtained height (dotted curve) and true height (solid curve) of acrylic bellshaped object

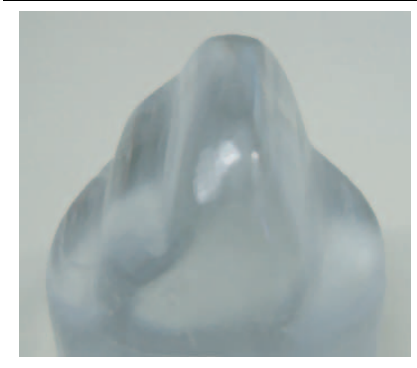

(a)

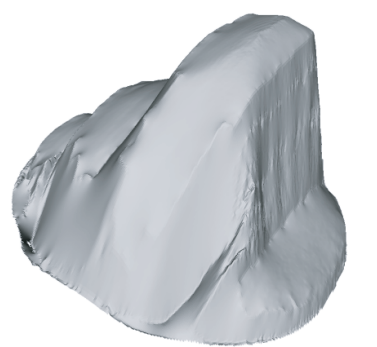

(b)
Figure 12. Measurement result of epoxy mountain-shaped object: (a) photograph of the object, (b) estimated shape

According to the figure, the height error was calculated to be approximately $0.4 \mathrm{~mm}$.

We also measured the object shown in figure 12(a) which is a mountain-shaped epoxy object. The refractive index of the object is 1.6 , and the diameter(width) and the height is $45 \mathrm{~mm}$ and $25 \mathrm{~mm}$ respectively. Figure 12 (b) shows the estimated shape of the object.

\section{Future work}

Error in our estimation is mainly caused by interreflection of transparent objects. Our future work is to develop a method which can handle the influence of interreflection.

\section{References}

[1] W. Heidrich, H. Lensch, M.F. Cohen, H-S Seidel, "Light Field Techniques for Reflections and Refractions," Proc. Eurographics Workshop on Rendering, pp.187-196, 1999.

[2] D.E. Zongker, D.M. Warner, B. Curless, D.H. Salesin, "Environmental matting and compositing," Proc. SIGGRAPH 99, pp.205-214, 1999.

[3] Y. Schechner, J. Shamir, N. Kiryuati, "Polarization-based decorrelation of transparent layers: the inclination angle of an invisible surface," Proc. IEEE Intl. Conf. Computer Vision, pp.814819, 1999.
[4] R. Szeliski, S. Avidan, P. Anandan, "Layer extraction from multiple images containing reflections and transparency," Proc. IEEE Conf. Computer Vision and Pattern Recognition, pp.246$253,2000$.

[5] H. Murase, "Surface shape reconstruction of an undulating transparent object," Proc. IEEE Intl. Conf. Computer Vision, pp.313-317, 1990.

[6] S. Hata, Y. Saitoh, S. Kumamura, K. Kaida, "Shape extraction of transparent object using genetic algorithm," Proc. Intl. Conf. Pattern Recognition, pp.684-688, 1996.

[7] K. Koshikawa, "A polarimetric approach to shape understanding of glossy objects," Proc. Intl. Joint Conf. Artificial Intelligence, pp.493-495, 1979.

[8] K. Koshikawa, Y. Shirai, "A model-based recognition of glossy objects using their polarimetrical properties," Advanced Robotics, 2(2), pp.137-147, 1987.

[9] L.B. Wolff, "Polarization-based material classification from specular reflection," IEEE Trans. Patt. Anal. Mach. Intell., 12(11), pp.1059-1071, 1990.

[10] L.B. Wolff, T.E. Boult, "Constraining object features using a polarization reflectance model," IEEE Trans. Patt. Anal. Mach. Intell., 13(7), pp.635-657, 1991.

[11] M. Saito, Y. Sato, K. Ikeuchi, H. Kashiwagi, "Measurement of surface orientations of transparent objects by use of polarization in highlight," J. Opt. Soc. Am. A, 16(9), pp.2286-2293, 1999.

[12] D. Miyazaki, M. Saito, Y. Sato, K. Ikeuchi, "Determining surface orientations of transparent objects based on polarization degrees in visible and infrared wavelengths," J. Opt. Soc. Am. A, 19(4), pp.687-694, 2002.

[13] S. Rahmann, N. Canterakis, "Reconstruction of specular surfaces using polarization imaging," Proc. IEEE Conf. Computer Vision and Pattern Recognition, pp.I:149-155, 2001.

[14] D. Miyazaki, R.T. Tan, K. Hara, K. Ikeuchi, "Polarizationbased Inverse Rendering from a Single View," Proc. IEEE Intl. Conf. Computer Vision, 2003. to appear

[15] M.P. do Carmo, Differential geometry of curves and surfaces, Prentice-Hall, 1976.

[16] B.K.P. Horn, Robot vision, MIT Press, 1986.

[17] J.J. Koenderink, Solid shape, MIT Press, 1990.

[18] M. Born, E. Wolf, Principles of optics, Pergamon Press, 1959.

[19] K. Ikeuchi, "Reconstructing a depth map from intensity maps," Proc. Intl. Conf. Pattern Recognition, pp.736-738, 1984. 